\title{
CONSTRUCTING METRICS ON A 2-TORUS WITH A PARTIALLY PRESCRIBED STABLE NORM
}

\author{
ERAN MAKOVER, HUGO PARLIER ${ }^{\ddagger}$, AND CRAIG J. SUTTON $\sharp$
}

\begin{abstract}
A result of Bangert states that the stable norm associated to any Riemannian metric on the 2-torus $T^{2}$ is strictly convex. We demonstrate that the space of stable norms associated to metrics on $T^{2}$ forms a proper dense subset of the space of strictly convex norms on $\mathbb{R}^{2}$. In particular, given a strictly convex norm $\|\cdot\|_{\infty}$ on $\mathbb{R}^{2}$ we construct a sequence $\left\langle\|\cdot\|_{j}\right\rangle_{j=1}^{\infty}$ of stable norms that converge to $\|\cdot\|_{\infty}$ in the topology of compact convergence and have the property that for each $r>0$ there is an $N \equiv N(r)$ such that $\|\cdot\|_{j}$ agrees with $\|\cdot\|_{\infty}$ on $\mathbb{Z}^{2} \cap\left\{(a, b): a^{2}+b^{2} \leq r\right\}$ for all $j \geq N$. Using this result, we are able to derive results on multiplicities which arise in the minimum length spectrum of 2-tori and in the simple length spectrum of hyperbolic tori.
\end{abstract}

\section{INTRODUCTION}

Given a closed $n$-dimensional manifold $M$ with first Betti-number $b=b_{1}(M)$, we let $H_{1}(M ; \mathbb{Z})_{\mathbb{R}}$ denote the collection of integral classes in the $b$-dimensional real vector space $H_{1}(M ; \mathbb{R})$. Then $H_{1}(M ; \mathbb{Z})_{\mathbb{R}}$ is a co-compact lattice in $H_{1}(M ; \mathbb{R})$. Letting $T \simeq \mathbb{Z}_{m_{1}} \times \cdots \times \mathbb{Z}_{m_{q}}$ denote the torsion subgroup of $H_{1}(M ; \mathbb{Z}) \simeq \mathbb{Z}^{b} \times T$, we see that $H_{1}(M ; \mathbb{Z})_{\mathbb{R}}$ can be identified with $H_{1}(M ; \mathbb{Z}) / T$ via the surjective homomorphism $\phi: H_{1}(M ; \mathbb{Z}) \rightarrow H_{1}(M ; \mathbb{Z})_{\mathbb{R}}$ given by

$$
\sum_{i=1}^{b} z_{i} h_{i}+t \mapsto\left(\sum_{i=1}^{b} z_{i} h_{i}\right) \otimes_{\mathbb{Z}} 1
$$

where $\left\{h_{1}, \ldots, h_{b}\right\}$ is some $\mathbb{Z}$-basis for $H_{1}(M ; \mathbb{Z})$, the $z_{i}$ 's are integers and $t \in T$. Now, let $\Psi: \pi_{1}(M) \rightarrow H_{1}(M ; \mathbb{Z})$ denote the Hurewicz homomorphism $[\mathbf{L}$, then the regular covering $p_{\text {Abel }}: M_{\text {Abel }} \rightarrow M$ of $M$ corresponding to $\operatorname{ker}(\Psi)=\left[\pi_{1}(M), \pi_{1}(M)\right]$ is the universal abelian covering of $M$. It is universal in the sense that it covers any other normal covering for which the deck transformations form an abelian group. The universal torsion-free abelian cover $p_{\text {tor }}: M_{\text {tor }} \rightarrow M$ corresponds to the normal subgroup $\Psi^{-1}(T) \triangleleft \pi_{1}(M)$ : it covers all other normal coverings for which the group of deck transformations is torsion-free and abelian. Under the above identifications we see that the group of deck transformations of $M_{\text {tor }} \rightarrow M$ is given by the lattice $H_{1}(M ; \mathbb{Z})_{\mathbb{R}}$. If $M$ has positive first Betti number, then to each metric $g$ we may associate a geometrically significant norm $\|\cdot\|_{s}$ on $H_{1}(M ; \mathbb{R})$ in the following manner.

For each $h \in H_{1}(M ; \mathbb{Z})_{\mathbb{R}} \simeq \mathbb{Z}^{b} \leq H_{1}(M ; \mathbb{R})$ let

$$
f(h)=\inf \left\{L_{g}(\sigma): \sigma \text { is a smooth loop representing the class } h\right\},
$$

1991 Mathematics Subject Classification. 53C20, 53C22.

Key words and phrases. stable norm, length spectrum.

${ }^{\ddagger}$ Research partially supported by Swiss National Science Foundation grant number PP00P2_128557.

\#Research partially supported by National Science Foundation grant DMS 0605247 and a Career Enhancement Fellowship from the Woodroow Wilson National Fellowship Foundation. 
where $L_{g}$ is the length functional associated to the Riemannian metric $g$ on $M$. Then for each $n \in \mathbb{N}$ we let $f_{n}: \frac{1}{n} H_{1}(M ; \mathbb{Z})_{\mathbb{R}} \rightarrow \mathbb{R}_{\geq 0}$ be given by

$$
f_{n}(h)=\frac{1}{n} f(n h) .
$$

It can be seen that the $f_{n}$ 's converge uniformly on comapct sets to a norm $\|\cdot\|_{s}$ on $H_{1}(M ; \mathbb{R})$ that is known as the stable norm of $g$ [B1]. In particular, if $\left\{v_{n}\right\}_{n \in \mathbb{N}}$ is a sequence in $H_{1}(M ; \mathbb{Z})_{\mathbb{R}}$ such that $\lim _{n \rightarrow \infty} \frac{v_{n}}{n}=v \in H_{1}(M ; \mathbb{R})$, then

$$
\|v\|_{s}=\lim _{n \rightarrow \infty} \frac{f\left(v_{n}\right)}{n} .
$$

An integral class $v \in H_{1}(M ; \mathbb{Z})_{\mathbb{R}}$ is said to be stable if there is an $n \in \mathbb{N}$ such that $\|v\|_{s}=$ $f_{n}(v)=\frac{f(n v)}{n}$. Intuitively, the stable norm $\|\cdot\|_{s}$ describes the geometry of the universal torsion-free abelian cover $\left(M_{\text {tor }}, g_{\text {tor }}\right)$ in a manner where the fundamental domain of the

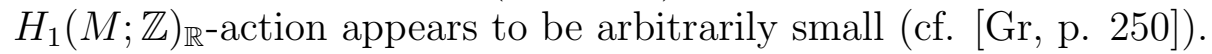

Now, let $p:(N, h) \rightarrow(M, g)$ be a Riemannian covering. We will say that a non-constant geodesic $\gamma: \mathbb{R} \rightarrow(M, g)$ is $p$-minimal (or minimal with respect to $p$ ) if for some and, hence, every lift $\tilde{\gamma}: \mathbb{R} \rightarrow N$ of $\gamma$, the geodesic $\tilde{\gamma}$ is distance minimizing between any two of its points. That is, $\gamma$ is $p$-minimal if for any $t_{1} \leq t_{2}$ we have $d_{N}\left(\tilde{\gamma}\left(t_{1}\right), \tilde{\gamma}\left(t_{2}\right)\right)=L_{g}(\tilde{\gamma} \uparrow$ $\left.\left[t_{1}, t_{2}\right]\right)$. In the event that $p$ is the universal Riemannian covering we will refer to $p$-minimal geodesics as minimal, and when $\gamma$ is minimal with respect to the universal abelian cover $p_{\text {abel }}:\left(M_{\text {Abel }}, h\right) \rightarrow(M, g)$ we will say that $\gamma$ is an abelian minimal geodesic. In the case where $\pi_{1}(M)$ is abelian-e.g., $M$ is a torus - these two definitions coincide.

An interesting application of the stable norm $\|\cdot\|_{s}$ is that characteristics of its unit ball $B \subset H_{1}\left(T^{2} ; \mathbb{Z}\right)$ can be used to deduce the existence (and properties) of minimal abelian geodesics. For instance, we have the following result due to Bangert.

Theorem 1.1 ([B1] Theorem 4.4 \& 4.8). Let $(M, g)$ be a Riemannian manifold and let $B \subseteq H_{1}(M ; \mathbb{R})$ be the unit ball corresponding to its stable norm. For every supporting hyperplane $H$ of $B$ there is an abelian minimal geodesic $\gamma: \mathbb{R} \rightarrow(M, g)$. As a consequence, $(M, g)$ has at least $k \equiv \operatorname{dim} H_{1}(M ; \mathbb{R})$ geometrically distinct abelian minimal geodesics.

In light of the relationship between the existence of minimal geodesics and the unit ball of the stable norm, it is an interesting question to determine which norms on $H_{1}(M ; \mathbb{R})$ arise as the stable norm associated to a Riemannian metric on $M$. In the case of the two-torus Bangert has made the following observation.

Theorem 1.2 ([B1] p. 267, [B2] Sec. 5). The collection of stable norms on $T^{2}$, denoted $\mathcal{N}_{\text {stab }}\left(T^{2}\right)$, is a proper subset of the collection of strictly convex norms on $\mathbb{R}^{2}$, denoted by $\mathcal{N}_{+}\left(\mathbb{R}^{2}\right)$.

Indeed, for any metric $g$ on a 2-torus we have that $f(k h)=|k| f(h)$ for any $k \in \mathbb{Z}$ and $h \in H_{1}(M ; \mathbb{Z})_{\mathbb{R}}$. Therefore, $\|h\|_{s}=f(h)$ on $H_{1}(M ; \mathbb{Z})_{\mathbb{R}}$. Now, suppose $h_{1}, h_{2} \in H_{1}(M ; \mathbb{Z})_{\mathbb{R}}$ are rationally independent and are represented by shortest geodesics $\gamma_{1}$ and $\gamma_{2}$ respectively. Then $\gamma_{1}$ and $\gamma_{2}$ must intersect transversally at $\gamma_{1}(0)=\gamma_{2}(0)$, for instance, and we conclude that $\gamma_{1} * \gamma_{2}$ is not smooth. Therefore, since the non-smooth curve $\gamma_{1} * \gamma_{2}$ represents the integral homology class $h_{1}+h_{2}$ we obtain the following strict inequality

$$
\left\|h_{1}+h_{2}\right\|_{s}<L_{g}\left(\gamma_{1} * \gamma_{2}\right)=L_{g}\left(\gamma_{1}\right)+L_{g}\left(\gamma_{2}\right)=\left\|h_{1}\right\|_{s}+\left\|h_{2}\right\|_{s} .
$$


It then follows that $\|\cdot\|_{s}$ is strictly convex norm on $H_{1}\left(T^{2}, \mathbb{R}\right) \simeq \mathbb{R}^{2}$. To see that $\mathcal{N}_{\text {stab }}\left(T^{2}\right)$ is a proper subset of $\mathcal{N}_{+}\left(\mathbb{R}^{2}\right)$, we recall that Bangert observed that on $T^{2}$ the stable norm is differentiable at irrational points [B2, Sec. 5]. That is, the unit ball of a stable norm associated to a Riemannian metric on $T^{2}$ has a unique supporting line at points $(x, y)$ where $y / x$ is irrational. But, one can readily see that there are many strictly convex norms which are not differentiable at such points. For instance, one need only take a strictly convex norm for which the unit ball is a tear drop whose singularity is placed at $(x, y)$ with $y / x$ irrational. And we conclude that $\mathcal{N}_{\text {stab }}\left(T^{2}\right)$ is a proper subset of $\mathcal{N}_{+}\left(\mathbb{R}^{2}\right)$.

In this article we will be concerned with stable norms of Riemannian 2-tori; henceforth referred to as toral stable norms. We show that the toral stable norms form a dense proper subset in the collection of all strictly convex norms on $H_{1}\left(T^{2} ; \mathbb{R}\right) \simeq \mathbb{R}^{2}$. Specifically, we demonstrate the following.

Theorem 1.3. Let $\|\cdot\|_{\infty}$ be a strictly convex norm on $H_{1}\left(T^{2} ; \mathbb{R}\right)$ and let $\left\langle h_{j} \equiv\left(a_{j}, b_{j}\right)\right\rangle_{j=1}^{\infty}$ be a sequence consisting of all of the integral homology classes $H_{1}(M ; \mathbb{Z})_{\mathbb{R}} \simeq \mathbb{Z}^{2}$ where $\left\|\left(a_{j}, b_{j}\right)\right\|_{\infty} \leq\left\|\left(a_{j+1}, b_{j+1}\right)\right\|_{\infty}$ for each $j$. Then there exists a sequence $\left\langle\|\cdot\|_{j}\right\rangle_{j=1}^{\infty}$ of toral stable norms such that

(i) for each $k \in \mathbb{N}$ we have $\left\|\left(a_{j}, b_{j}\right)\right\|_{k}=\left\|\left(a_{j}, b_{j}\right)\right\|_{\infty}$ for $1 \leq j \leq k$, while $\left\|\left(a_{j}, b_{j}\right)\right\|_{k} \geq$ $\left\|\left(a_{k}, b_{k}\right)\right\|_{\infty}$ for all $j \geq k+1$

(ii) $\lim _{j \rightarrow \infty}\|\cdot\|_{j}=\|\cdot\|_{\infty}$ in the topology of compact convergence.

Hence, any strictly convex norm on $\mathbb{R}^{2}$ can be approximated uniformly on compact sets by a stable norm that agrees with it on an arbitrarily large set of lines through the origin with rational slope. We now show that this result can be interpreted in terms of the minimum marked length spectrum of a torus.

First, we recall that the length spectrum of a Riemannian manifold $(M, g)$ is the collection of lengths of all smoothly closed geodesics in $(M, g)$, where we adopt the convention that the multiplicity of a length $\ell$ is counted according to the number of free homotopy classes containing a geodesic of that length. Now, given a loop $\sigma$ on a manifold $M$ its unoriented free homotopy class is the collection of closed geodesics that are freely homotopic to $\sigma$ or its inverse $\bar{\sigma}$. We will denote the collection of the unoriented free homotopy classes by $\mathcal{F}(M)$ and let $\pi: \pi_{1}(M) \rightarrow \mathcal{F}(M)$ denote the natural projection. We then define the minimum length spectrum to be the (possibly finite) sequence $\ell_{1}=0<\ell_{1} \leq \ell_{2} \leq \cdots$ consisting of the lengths of closed geodesics that are shortest in their unoriented free homotopy class, where a length $\ell$ is repeated according to the number of unoriented free homotopy classes whose shortest geodesic is of length $\ell$. If we wish to keep track of the unoriented free homotopy classes we then consider the map $m_{g}: \mathcal{F}(M) \rightarrow \mathbb{R}$ which assigns to each unoriented free homotopy class the length of its shortest closed geodesic. We will refer to $m_{g}$ or the collection $\left\{\left(m_{g}(\alpha), \alpha\right): \alpha \in \mathcal{F}(M)\right\}$ as the minimum marked length spectrum of $(M, g)$ (see [DGS, Def. $2.8])$.

It is natural to ask which pairs $(\ell, \alpha)$ consisting of a nonnegative number $\ell$ and an unoriented free homotopy class $\alpha$ can occur as part of the mimium marked length spectrum associated to some metric $g$ on $M$. This question was addressed in dimension three and higher by the third author, along with De Smit and Gornet, in [DGS] where the following was shown.

Theorem 1.4 ([DGS] Theorem 2.9). Suppose that $M$ is a closed connected manifold of dimension at least three. Let $\alpha=\left(\alpha_{1}, \alpha_{2}, \ldots, \alpha_{k}\right)$ be a sequence of distinct elements of 
$\mathcal{F}(M)$ where $\alpha_{1}$ is trivial. Then for every sequence $0=\ell_{1}<\ell_{2} \leq \cdots \leq \ell_{k}$ of real numbers the following are equivalent:

(i) The sequence $\ell_{1}, \ldots, \ell_{k}$ is $\alpha$-admissible; that is, for $i, j=2, \ldots, k \ell_{i} \leq|n| \ell_{j}$, whenever $\alpha_{i}=\alpha_{j}^{n}$ for some $n \in \mathbb{Z}$ and for $i=2, \ldots, k \ell_{i} \geq \frac{1}{|n|} \ell_{k}$ whenever $\alpha_{i}^{n} \notin\left\{\alpha_{1}, \ldots, \alpha_{k}\right\}$ for some $n \neq 0 \in \mathbb{Z}$.

(ii) There is a Riemannian metric $g$ on $M$ such that the minimum marked length spectrum $m_{g}: \mathcal{F}(M) \rightarrow \mathbb{R}_{\geq 0}$ satisfies $m_{g}\left(\alpha_{i}\right)=\ell_{i}$ for all $i$ and $m_{g}(\alpha) \geq \ell_{k}$ for all $\alpha \in \mathcal{F}(M)-$ $\left\{\alpha_{1}, \ldots, \alpha_{k}\right\}$.

In particular, there is a metric $g$ on $M$ such that the systole is achieved in the unoriented free homotopy class $\alpha_{2}$.

The proof of Theorem 1.4 depends on the fact that a finite collection of distinct unoriented free homotopy classes can be represented by pairwise disjoint simple closed curves. The fact that this does not hold in dimension two appears to make approaching this question for surfaces - the actual motivation behind this article - a more delicate matter. However, we note that among surfaces the torus enjoys some special properties. First, all free homotopy classes can be represented by a simple closed curve or an iterate of such a curve. Consequently, with respect to any metric, the shortest closed geodesic in a free homotopy class will be a simple closed curve if the class is primitive, or an iterate of a simple closed curve in the case of a non-primitive class. Secondly, it follows from the fact that $T^{2}$ is an aspherical surface that for any choice of smooth Riemannian metric $g$ and choice of non-trivial free homotopy class $[\beta]$, a closed geodesic of minimal length in $[\beta]$ will have a minimal number of self-intersections [FHS]. In Section 2, these properties will be marshaled to prove Theorem 1.3(i) which in conjunction with Bangert's Theorem 1.2 gives the following statement concerning the minimum marked length spectrum of a 2-torus.

Theorem 1.5. Let $T^{2}$ be a 2-torus and $\pi_{1}\left(T^{2}\right) \simeq \mathbb{Z}^{2} \leq \mathbb{R}^{2}$ its fundamental group. Now, let $\alpha=\left(\alpha_{1}, \ldots, \alpha_{k}\right)$ be a sequence of distinct unoriented free homotopy classes of $T^{2}$, where $\alpha_{i}$ is represented by $\pm\left(a_{i}, b_{i}\right) \in \mathbb{Z}^{2}$ and $\alpha_{1}=(0,0)$ is trivial. Also, let $\ell_{1}=0<\ell_{2} \leq \cdots \leq \ell_{k}$ be a finite sequence. Then the following are equivalent:

(i) There is a strictly convex norm $\|\cdot\|$ on $\mathbb{R}^{2}$ such that $\left\|\left(a_{i}, b_{i}\right)\right\|=\ell_{i}$ and $\|(a, b)\| \geq \ell_{k}$ for any $(a, b) \neq \pm\left(a_{1}, b_{1}\right), \ldots, \pm\left(a_{k}, b_{k}\right)$.

(ii) There is a metric $g$ on $T^{2}$ such that the minimum marked length spectrum $m_{g}$ : $\mathcal{F}\left(T^{2}\right) \rightarrow \mathbb{R}_{\geq 0}$ satisfies $m_{g}\left(\alpha_{i}\right)=\ell_{i}$ for all $i=1, \ldots, k$ and $m_{g}(\alpha) \geq \ell_{k}$ for all $\alpha \in \mathcal{F}\left(T^{2}\right)-\left\{\alpha_{1}, \ldots, \alpha_{k}\right\}$.

In Section 4 we consider the multiplicities in the minimum length spectrum of a 2-torus. By using results concerning the minimum number of lattice points in the interior of an $n$-gon and Theorem 1.3(i) we obtain the following estimate on the "location" of a length with a specified multiplicity.

Theorem 1.6. Suppose $\left(T^{2}, g\right)$ is a torus for which the minimum length spectrum $\left\langle\ell_{j}\right\rangle_{j=1}^{\infty}$ has a length of multiplicity $m$. That is, for some $n \in \mathbb{N}$ we have $0=\ell_{1} \leq \ell_{n}<\ell_{n+1}=\cdots=$ $\ell_{n+m}<\ell_{n+m+1}$. Then $n=m_{g}^{-1}([0, \ell))=f(m) \equiv \frac{i_{0}^{\text {symm }}(2 m)+1}{2} \geq O\left(m^{3}\right)$, where $i_{0}^{\text {symm }}(2 m)$ is the minimum number of integer points in the interior of a convex integer $2 m$-gon that is centrally symmetric with respect to $(0,0)$. Furthermore, this inequality is sharp. That is, for each $m \in \mathbb{N}$ there is a smooth metric $g$ on $T^{2}$ and $\ell>0$ such that $\ell$ has multiplicity $m$ in the minimum length spectrum and $m_{g}^{-1}([0, \ell))=f(m)$. 
Our study of the multiplicities of the minimum length spectrum of a torus is motivated in part by the study of hyperbolic surfaces; especially, hyperbolic punctured tori. The length spectrum of a hyperbolic surface always contains lengths of arbitrarily high multiplicity $[\mathrm{Ran}$, and any closed geodesic is of minimal length on a hyperbolic surface. Unlike the case of smooth tori, hyperbolic surfaces contain non-simple closed geodesics which are thus minimal in their homotopy class, and it is among these geodesics that high multiplicities are known to appear. To date, multiplicities have not been observed among the simple closed geodesics and it is a conjecture of Schmutz Schaller that among primitive simple closed geodesics on a once-punctured torus the multiplicity of a given length is bounded by 6. This conjecture is a specific case of a more general conjecture, due to Rivin, asserting that multiplicity in the simple length spectrum - the collection of lengths of simple closed geodesics - is always bounded by a constant that only depends on the underlying topology (see [Sch, p. 209]).

Presently, not much is known about the validity of the conjectures of Schmutz Schaller and Rivin. However, Theorem 1.6 gives new examples demonstrating that these conjectures do not hold for arbitrary surfaces; in particular, tori (cf. [MP, p. 1884-5]). We note that Theorem 1.6 can be used to relate the multiplicity of the length $\ell$ to its position in the simple length spectrum of a one-holed or once-punctured torus.

Corollary 1.7. If there are $m$ simple closed geodesics of the same length $\ell$ on a oncepunctured (or one-holed) torus, then there are at least $f(m)$ distinct simple closed geodesics of length strictly less than $\ell$.

Unlike the conjectures of Schmutz Schaller and Rivin, the geodesics considered in Corollary 1.7 include geodesics representing non-primitive classes and the function $f(m)$ counts the trivial homology/homotopy class. Of course if Rivin's conjecture is correct, then Corollary 1.7 might only be of interest for small values of $m$.

\section{Constructing the Stable Norms: the Proofs of Theorems 1.3(i) and 1.5}

In this section we will prove Theorems $1.3(\mathbf{i})$ and 1.5 . The basic idea behind the proof of Theorem 1.3(i) is to isolate geodesics $\gamma_{1}, \gamma_{2}, \ldots, \gamma_{k}$ on a flat torus $\left(T^{2}, g_{0}\right)$, with a systole of at least $\ell_{k}$, representing the $k$ homology classes $h_{1}, h_{2}, \ldots, h_{k} \in H_{1}\left(T^{2}, \mathbb{Z}\right)$ in the statement of the Theorem and then dig deep "canyons" with narrow "corridors" of the appropriate length along these geodesics in order to obtain a new metric $g_{k}$ for which the conclusions of the theorem are obtained. Theorem 1.5 will then follow as an application of Theorem $1.3(\mathbf{i})$ and Bangert's result that the stable norm of a metric on a 2-torus is strictly convex.

2.1. The proof of Theorem $1.3(\mathbf{i})$. Let $\|\cdot\|_{\infty}$ denote a fixed strictly convex norm on $H_{1}\left(T^{2}, \mathbb{R}\right) \simeq \mathbb{R}^{2}$ and let $\left\langle h_{i}=\left(a_{i}, b_{i}\right)\right\rangle_{i=1}^{\infty}$ denote a fixed enumeration of the integral homology classes $H_{1}\left(T^{2}, \mathbb{Z}\right) \simeq \mathbb{Z}^{2}$ with the property that $\left\|h_{i}\right\|_{\infty} \leq\left\|h_{i+1}\right\|_{\infty}$ for each $i \in \mathbb{N}$. In this section we wish to show that for each $k \in \mathbb{N}$ we may find a toral stable norm $\|\cdot\|_{k}$ such that $\left\|h_{i}\right\|_{k}=\left\|h_{i}\right\|_{\infty}$ for $1 \leq i \leq k$, while $\left\|h_{j}\right\|_{k} \geq\left\|h_{k}\right\|_{\infty}$ for each $j \geq k+1$. We begin by fixing some notation and assumptions that will hold throughout this section.

\section{Notation, Assumptions \& Remarks 2.1.}

1. For any Riemannian metric $g$ on $T^{2}$ we let $L_{g}$ denote the length functional on the loop space and we let $d_{g}$ be the distance function in the induced metric space structure. 
2. For any loop $\sigma: S^{1} \rightarrow T^{2}$ we will let $\operatorname{Im}(\sigma)$ denote the image of $\sigma$ and we wil let $h_{\sigma} \equiv$ $\left(a_{\sigma}, b_{\sigma}\right) \in H_{1}\left(T^{2} ; \mathbb{Z}\right) \simeq \mathbb{Z}^{2}$ denote its homology class.

3. For each $i \in \mathbb{N}$ we will let $\ell_{i}=\left\|h_{i}\right\|_{\infty}$.

4. We will say that a homology class $h \in H_{1}\left(T^{2} ; \mathbb{Z}\right)$ is primitive if whenever $h=n \widetilde{h}$, for some $n \in \mathbb{N}$ and $\widetilde{h} \in H_{1}\left(T^{2} ; \mathbb{Z}\right)$, we have $n=1$ and $\widetilde{h}=h$.

5. Since for any norm $\|\cdot\|$ on a real vector space $\mathcal{V}$ we have $\|r v\|=|r|\|v\|$, where $r \in \mathbb{R}$ and $v \in \mathcal{V}$, we may assume without loss of generality that each $h_{i}=\left(a_{i}, b_{i}\right) \in H_{1}\left(T^{2} ; \mathbb{Z}\right)$ is a primitive homology class and that for $i \neq j$ we have $h_{i} \neq \pm h_{j}$.

6. When convenient we will identify a homology class $h \in H_{1}\left(T^{2} ; \mathbb{Z}\right)$ with the free homotopy class $\Psi^{-1}(h)$ given by the Hurewicz isomorphism $\Psi: \pi_{1}\left(T^{2}, p_{o}\right) \rightarrow H_{1}\left(T^{2} ; \mathbb{Z}\right)$, where $p_{0}$ is some fixed point in $T^{2}$.

7. We will let $g_{0}$ denote a fixed flat metric on $T^{2}$ with systole satisfying $\operatorname{Syst}\left(T^{2}, g_{0}\right) \geq \ell_{k}$ and set $B \equiv \operatorname{Syst}\left(T^{2}, g_{0}\right)$.

8. For each $i \in \mathbb{N}$ we will let $\gamma_{i}$ be the unique geodesic in $\left(T^{2}, g_{0}\right)$ passing through $p_{0}$ and representing the primitive homology class $h_{i}$. We note that since $h_{1}=(0,0)$ represents the trivial class, the geodesics $\gamma_{1}$ is trivial.

9. Theorem 1.3(i) is then equivalent to showing that for each $k \in \mathbb{N}$ there is a metric $g_{k}$ such that

(a) $L_{g_{k}}\left(\gamma_{i}\right)=\ell_{i}$,

(b) for any loop $\sigma$ in $\left(T^{2}, g_{k}\right)$, representing one of the (primitive) homology classes $\left\{h_{i}\right\}_{i \in \mathbb{N}}$, we have

$$
L_{g_{k}}(\sigma) \geq \begin{cases}\ell_{i} & h_{\sigma}=h_{i} \text { for some } i=1, \ldots, k \\ \ell_{k} & \text { otherwise }\end{cases}
$$

10. By a cycle $c$ in a graph $\mathcal{G}$ we will mean a sequence of vertices $\left\langle v_{i}\right\rangle_{i=0}^{q}$ such that $v_{0}=v_{q}$ and for each $i=0,1, \ldots, q-1$ there is an edge $e_{i}$ joining $v_{i}$ and $v_{i+1}$. The edge length of such a cycle is said to be $q$.

11. It is clear that if $(\mathcal{G}, d)$ is a metric graph, then for any loop $\sigma: S^{1} \rightarrow \mathcal{G}$ there is a cycle $c$ that is freely homotopic to $\sigma$ in $\mathcal{G}$ such that $L(\sigma) \geq L(c)$. A cycle $c$ will be said to be minimal if it is the shortest cycle in its free homotopy class. Clearly a minimal cycle will have minimal edge length among all other cycles in its free homotopy class.

Fix $k \in \mathbb{N}$ and let $h_{1}=\left(a_{1}, b_{1}\right), \ldots, h_{k}=\left(a_{k}, b_{k}\right) \in H_{1}\left(T^{2} ; \mathbb{Z}\right)$ be the first $k$ homology classes in our ordering. Since $T^{2}$ is a torus we see that for each $2 \leq i \neq j \leq k$ the geodesics $\gamma_{i}$ and $\gamma_{j}$ intersect transversally in finitely many points. Consider the curves $\gamma_{1}, \gamma_{2}, \ldots, \gamma_{k}$ simultaneously and let $\left\{p_{0}, p_{1}, \ldots, p_{t}\right\}$ be the collection of intersection points. Then for each $i=2, \ldots, k$ these points partition $\gamma_{i}$ into $m_{i}$ segments $\gamma_{i 1}, \ldots, \gamma_{i m_{i}}$, and since $g_{0}$ is a flat metric on $T^{2}$ one can deduce that the quantity $q_{i j} \equiv \frac{L_{g_{0}}\left(\gamma_{i j}\right)}{L_{g_{0}}\left(\gamma_{i}\right)}$ is a positive rational number, for each $i=2, \ldots, k$ and $1 \leq j \leq m_{i}$. The union of the images of the geodesics $\gamma_{1}, \gamma_{2}, \ldots, \gamma_{k}$, which we will denote by $\mathcal{G}$, forms a directed graph in $T^{2}$, where the points $\left\{p_{0}, p_{1}, \ldots, p_{t}\right\}$ are the vertices and the segments $\gamma_{i j}$ are the oriented edges. Now suppose $\mathcal{T}$ is a regular neighborhood of $\mathcal{G}$ with smooth boundary in $T^{2}$ (see Figure 1). Then $\mathcal{T}$ can be decomposed into $t+1$ disjoint "hubs" $\left\{\Delta_{0}, \Delta_{1}, \ldots, \Delta_{t}\right\}$ containing the vertices $\left\{p_{0}, p_{1}, \ldots, p_{t}\right\}$ and disjoint (rectangular) "corridors" $R_{i j}$ containing $\operatorname{Im}\left(\gamma_{i j}\right)-\cup_{s=0}^{t} \Delta_{s}$ (see Figure 2). We now show that we can find a regular neighborhood $\mathcal{T}$ of $\mathcal{G}$ and a flat metric $\rho_{1}$ defined on $\mathcal{T}$ such that 
Theorem 1.3(i) - in the guise of 2.1 (ㄱ) above - is true if we restrict our attention to loops contained in $\left(\mathcal{T}, \rho_{1}\right)$. Specifically, we have the following lemma.

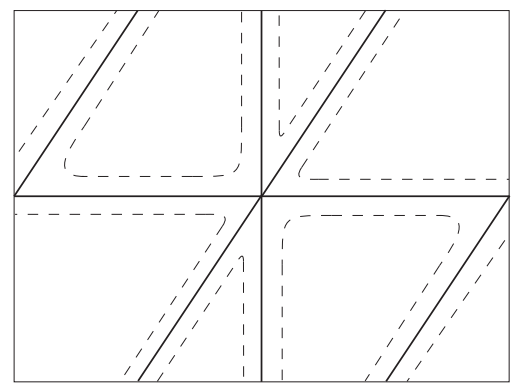

FiguRE 1. Regular Neighborhood of $\mathcal{G}$

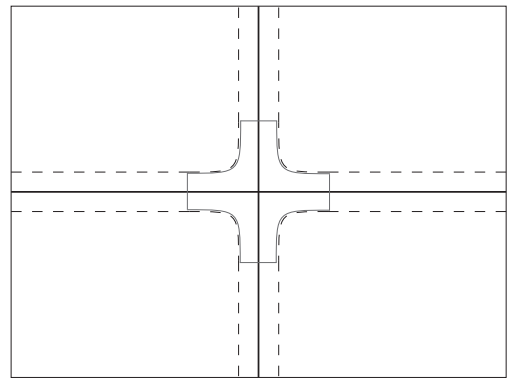

Figure 2. Decomposition into "hubs" \& "corridors"

Lemma 2.2. With the notation as above, there is a regular neighborhood $\mathcal{T}$ of $\mathcal{G}$ with smooth boundary and a flat metric $\rho_{1}$ on $\mathcal{T}$ with the following properties:

(1) $L_{\rho_{1}}\left(\gamma_{i}\right)=\ell_{i}$ for $i=1,2, \ldots, k$;

(2) if $\sigma$ is a loop in $\mathcal{T}$ representing the (primitive) homology class $\left(a_{\sigma}, b_{\sigma}\right) \in H_{1}\left(T^{2}, \mathbb{Z}\right) \simeq \mathbb{Z}^{2}$, then

$$
L_{\rho_{1}}(\sigma) \geq \begin{cases}\ell_{i} & \left(a_{\sigma}, b_{\sigma}\right)= \pm\left(a_{i}, b_{i}\right) \text { for some } i=1, \ldots, k \\ \ell_{k} & \text { otherwise }\end{cases}
$$

Proof of Lemma 2.2. The proof of this lemma has three main steps:

(A) We take an arbitrary regular neighborhood $\mathcal{T}^{\prime}$ of $\mathcal{G}$ with a particular choice of "hubs" $\left\{\Delta_{0}, \Delta_{1}, \ldots, \Delta_{t}\right\}$ and corresponding rectangular "corridors" $\left\{R_{i j}: i=2, \ldots, k, j=\right.$ $\left.1, \ldots, m_{i}\right\}$. Then we adjust the length of the corridors to obtain a flat metric $\rho_{1}$ on $\mathcal{T}^{\prime}$ for which condition (1) is satisfied and $L_{\rho_{1}}\left(\gamma_{i j}\right)=q_{i j} \ell_{i}$, where we recal that the $q_{i j}$ 's are rational.

(B) We use the strict convexity of the norm $\|\cdot\|_{\infty}$ and the fact that $L_{\rho_{1}}\left(\gamma_{i j}\right)=q_{i j} \ell_{i}$ for $q_{i j} \in \mathbb{Q}$ to show that for any minimal cycle $c$ in the length space $\left(\mathcal{G}, \rho_{1}\right)$ that is not a reparametrization of $\gamma_{1}, \gamma_{2}, \ldots, \gamma_{k}$ the following strict inequality holds:

$$
L_{\rho_{1}}(c)>\left\|\left(a_{c}, b_{c}\right)\right\|_{\infty} .
$$

Then, since $\left\|\left(a_{i}, b_{i}\right)\right\|_{\infty} \leq\left\|\left(a_{i+1}, b_{i+1}\right)\right\|_{\infty}$ for each $i$, we see that the lemma is true on the length space $\left(\mathcal{G}, \rho_{1}\right)$. 
(C) We use the inequality from the previous step to obtain a constant $\Theta>0$ with the property that if $\mathcal{T} \subset \mathcal{T}^{\prime}$ is a regular neighborhood of $\mathcal{G}$ with "hubs" $\left\{\Delta_{0}, \Delta_{1}, \ldots, \Delta_{t}\right\}$ satisfying

$$
\operatorname{diam} \Delta_{s} \leq \max _{x \in \partial \Delta_{s}} 2 \cdot d\left(p_{s}, x\right) \leq \Theta
$$

where the distance is computed with respect to $\rho_{1}$, then the lemma holds on $\left(\mathcal{T}, \rho_{1}\right)$.

Step A: Choose an arbitrary regular neighborhood $\mathcal{T}^{\prime}$ of $\mathcal{G}$ in $T^{2}$ and a collection of "hubs" $\left\{\Delta_{0}^{\prime}, \Delta_{1}^{\prime}, \ldots, \Delta_{t}^{\prime}\right\}$, with a corresponding collection of rectangular "corridors" $\left\{R_{i j}^{\prime}: 2 \leq i \leq\right.$ $\left.k, 1 \leq j \leq m_{i}\right\}$, having the property that

$$
L_{g_{0}}\left(\operatorname{Im}\left(\gamma_{i j}\right) \cap \cup_{s=0}^{t} \Delta_{s}\right)<\frac{1}{2} q_{i j} \ell_{i},
$$

for each $2 \leq i \leq k, 1 \leq j \leq m_{i}$. That is, each edge $\gamma_{i j}$ in our graph $\mathcal{G}$ has less than $\frac{1}{2} q_{i j} \ell_{i}$ of its length contained in the "hubs". Then by lengthening or shortening each $R_{i j}$ in the " $\gamma_{i j}$-direction" we obtain a new flat metric $\rho_{1}$ on $\mathcal{T}^{\prime}$ with respect to which we have $L_{\rho_{1}}\left(\gamma_{i j}\right)=q_{i j} \ell_{i}$ for $2 \leq i \leq k 1 \leq j \leq m_{i}$, and (recalling that $\gamma_{1}$ is trivial) we see that $L_{\rho_{1}}\left(\gamma_{i}\right)=\ell_{i}$, for $i=1,2, \ldots, k$. Hence, condition $(\mathbf{1})$ of the Lemma is obtained on $\left(\mathcal{T}^{\prime}, \rho_{1}\right)$. We note that the fact that $L_{\rho_{1}}\left(\gamma_{i j}\right)=q_{i j} \ell_{i}$ for each $i=2, \ldots, k$ and $j=1, \ldots, m_{i}$ will be exploited in Step B.

We now want to demonastrate that by picking a thin regular neighborhood $\mathcal{T} \subset \mathcal{T}^{\prime}$ of $\mathcal{G}$ where the "hubs" can be chosen of suffciently small diameter we can also obtain condition (2). Towards this end we first show that the lemma is true on our metric graph $\left(\mathcal{G}, \rho_{1}\right)$.

Step B: Let $c$ be a minimal cycle in $\left(\mathcal{G}, \rho_{1}\right)$ representing the homology class $\left(a_{c}, b_{c}\right) \in$ $H_{1}\left(T^{2}, \mathbb{Z}\right)$. Now, for each $i=2, \ldots, k$ and $j=1, \ldots, m_{i}$ we let $n_{i j}^{+}(c)\left(\operatorname{resp} . n_{i j}^{-}(c)\right)$ denote the number of times $c$ traverses the edge $\gamma_{i j}$ in the positive direction (respectively, negative direction). Then with respect to the metric $\rho_{1}$ the length of the curve $c$ is given by

$$
\begin{aligned}
L_{\rho_{1}}(c) & =\sum_{i=2}^{k} \sum_{j=1}^{m_{i}}\left(n_{i j}^{+}(c)+n_{i j}^{-}(c)\right) L_{\rho_{1}}\left(\gamma_{i j}\right) \\
& =\sum_{i, j}\left(n_{i j}^{+}(c)+n_{i j}^{-}(c)\right) q_{i j} \ell_{i} \\
& =\sum_{i, j}\left(n_{i j}^{+}(c)+n_{i j}^{-}(c)\right) q_{i j}\left\|\left(a_{i}, b_{i}\right)\right\|_{\infty} .
\end{aligned}
$$

Since each $q_{i j}$ is rational, we may fix $N \in \mathbb{N}$ so that $N q_{i j}$ is an integer for each $i=2, \ldots, k$ and $j=1, \ldots, m_{i}$. Then $c^{N}$ represents the homology class $N\left(a_{c}, b_{c}\right)$ and has length

$$
L_{\rho_{1}}\left(c^{N}\right)=\sum_{i=2}^{k} \sum_{j=1}^{m_{i}} N\left(n_{i j}^{+}(c)+n_{i j}^{-}(c)\right) q_{i j}\left\|\left(a_{i}, b_{i}\right)\right\|_{\infty} .
$$

That is, each edge $\gamma_{i j}$ contributes $N\left(n_{i j}^{+}(c)+n_{i j}^{-}(c)\right) q_{i j}\left\|\left(a_{i}, b_{i}\right)\right\|_{\infty}$ towards the length of $c^{N}$. Now, for each $i=2, \ldots, k$, let $N_{i}=\sum_{j=1}^{m_{i}} N\left(n_{i j}^{+}(c)-n_{i j}^{-}(c)\right) q_{i j}$. Then $\delta_{i} \equiv \gamma_{i}^{N_{i}}$ is a curve in $\mathcal{G}$ representing the homology class $N_{i}\left(a_{i}, b_{i}\right) \in H_{1}\left(T^{2} ; \mathbb{Z}\right)$. It then follows from the definition 
of the $n_{i j}^{+}(c)$ 's and $n_{i j}^{-}(c)$ 's that the curves $c^{N}$ and $\delta=\delta_{2} * \cdots * \delta_{k}$ in $\mathcal{G}$ have the same algebraic intersection number with a basis for $H_{1}\left(T^{2} ; \mathbb{Z}\right)$. Therefore, since a homology class in $H_{1}\left(T^{2} ; \mathbb{Z}\right)$ is determined by its algebraic intersection numbers with a basis for $H_{1}\left(T^{2} ; \mathbb{Z}\right)$, we conclude that $c^{N}$ and $\delta$ are homologous, and we obtain the following expression for $N\left(a_{c}, b_{c}\right)$ :

$$
\begin{aligned}
N\left(a_{c}, b_{c}\right) & =h_{c^{N}} \\
& =h_{\delta} \\
& =\sum_{i=2}^{k} N_{i}\left(a_{i}, b_{i}\right) \\
& =\sum_{i=2}^{k} \sum_{j=1}^{m_{i}} N\left(n_{i j}^{+}(c)-n_{i j}^{-}(c)\right) q_{i j}\left(a_{i}, b_{i}\right) .
\end{aligned}
$$

The strict convexity of the norm $\|\cdot\|_{\infty}$ allows us to obtain the following:

$$
\begin{aligned}
N\left\|\left(a_{c}, b_{c}\right)\right\|_{\infty} & =\left\|N\left(a_{c}, b_{c}\right)\right\|_{\infty} \\
& =\left\|\sum_{i=2}^{k} \sum_{j=1}^{m_{i}} N\left(n_{i j}^{+}(c)-n_{i j}^{-}(c)\right) q_{i j}\left(a_{i}, b_{i}\right)\right\|_{\infty} \\
& <\sum_{i=2}^{k} \sum_{j=1}^{m_{i}} N\left|\left(n_{i j}^{+}(c)-n_{i j}^{-}(c)\right)\right| q_{i j}\left\|\left(a_{i}, b_{i}\right)\right\|_{\infty} \\
& \leq \sum_{i=2}^{k} \sum_{j=1}^{m_{i}} N\left(n_{i j}^{+}(c)+n_{i j}^{-}(c)\right) q_{i j}\left\|\left(a_{i}, b_{i}\right)\right\|_{\infty} \\
& =N L_{\rho_{1}}(c) .
\end{aligned}
$$

Dividing through by $N$ in the inequality above we obtain

$$
L_{\rho_{1}}(c)>\left\|\left(a_{c}, b_{c}\right)\right\|_{\infty} .
$$

As it will be useful in the sequel, we pause to define the notions of hub length and corridor length for a loop $\sigma: S^{1} \rightarrow(\widetilde{\mathcal{T}}, \widetilde{\rho})$ in an arbitrary flat regular neighborhood of $\mathcal{G}$. Let $(\widetilde{\mathcal{T}}, \widetilde{\rho})$ be such a regular neighborhood with a corresponding choice of hubs $\left\{\widetilde{\Delta}_{0}, \widetilde{\Delta}_{1}, \ldots, \widetilde{\Delta}_{s}\right\}$ and rectangular corridors $\left\{\widetilde{R}_{i j}: i=2, \ldots, k, j=1,2, \ldots, m_{i}\right\}$. Then the corridor length of $\sigma$ is defined to be: $L_{\tilde{\rho}}^{\text {hub }}(\sigma) \equiv L_{\tilde{\rho}}\left(\operatorname{Im}(\sigma) \cap \cup_{s=0}^{t} \widetilde{\Delta}_{s}\right)$. Similarly, the corridor length of $\sigma$ is defined to be: $L_{\tilde{\rho}}^{\text {cor }}(\sigma)=L_{\tilde{\rho}}\left(\operatorname{Im}(\sigma) \cap \cup_{i, j} \widetilde{R}_{i j}\right)$. Due to the flatness of the metric $\tilde{\rho}$ and that the corridors are actually rectangles, it is clear that for any curve $\sigma$ in $(\tilde{\mathcal{T}}, \tilde{\rho})$ freely homotopic in $\tilde{\mathcal{T}}$ to a minimal cycle $c_{\sigma}$ in $\mathcal{G}$ that we have:

$$
L_{\tilde{\rho}}^{\mathrm{cor}}(\sigma) \geq L_{\tilde{\rho}}^{\mathrm{cor}}\left(c_{\sigma}\right) .
$$

Step C: We are now in a position to explain how to pick our regular neighborhood $\mathcal{T} \subset \mathcal{T}^{\prime}$. We begin by defining a particular collection of cycles in our graph $\mathcal{G}$. 
Let $\mathcal{C}$ denote the collection of minimal cycles $c$ in the length space $\left(\mathcal{G}, \rho_{1}\right)$ with the following properties:

(1) $c$ is not freely homotopic to the cycles $\gamma_{1}, \gamma_{2}, \ldots, \gamma_{k}$ in $\mathcal{T}^{\prime}$. (We note that this does not preclude $\left(a_{c}, b_{c}\right)=\left(a_{i}, b_{i}\right)$ for some $i=1,2, \ldots, k$.);

(2) $c$ consists of at most $\left\lfloor\frac{\ell_{k}}{\zeta}\right\rfloor$ edges, where $\zeta \equiv \frac{1}{2} \min \left\{q_{i 1} \ell_{i}, \ldots, q_{i m_{i}} \ell_{i}: i=1, \ldots, k\right\}$ and $\lfloor x\rfloor$ denotes the greatest integer less than $x>0$. (We note that it follows from Equation 2.3 and the manner in which the metric $\rho_{1}$ was constructed that the length of each rectangle $R_{i j}$ in the " $\gamma_{i j}$-direction" is greater than $\zeta$.)

It will prove to be useful to notice that the upper bound on edge length of elements of $\mathcal{C}$ implies that $\mathcal{C}$ is a finite collection. It now follows from Equation 2.4 that the quantity

$$
\tilde{\epsilon} \equiv \min _{c \in \mathcal{C}}\left(L_{\rho_{1}}(c)-\left\|\left(a_{c}, b_{c}\right)\right\|_{\infty}\right)
$$

is positive.

Now let $\sigma$ be a curve in the tubular neighborhood $\left(\mathcal{T}^{\prime}, \rho_{1}\right)$ that is freely homotopic in $\mathcal{T}^{\prime}$ to the minimal cycle $c_{\sigma} \in \mathcal{C}$ of edge length $q \leq\left\lfloor\frac{\ell_{k}}{\zeta}\right\rfloor$. The edges of $c_{\sigma}$ determine $q$ corridors $R_{1}, \ldots, R_{q}$ through which it passes (counted with multiplicity). Then $\sigma$ must pass through these $q$ corridors. In fact, since we are ultimately interested in obtaining a lower bound on the length of $\sigma$, we may assume without loss of generality that $\sigma$ enter and leaves precisely these $q$ corridors (counting multiplicities) and no other corridors. As noted earlier, since $\left(\mathcal{T}^{\prime}, \rho_{1}\right)$ is flat we see that $L_{\rho_{1}}^{\text {cor }}(\sigma) \geq L_{\rho_{1}}^{\text {cor }}\left(c_{\sigma}\right)$. Hence, the only way that $\sigma$ can be shorter than $c_{\sigma}$ is to "make up the difference" inside the "hubs"; that is, we need the quantity $L_{\rho_{1}}^{\mathrm{hub}}\left(c_{\sigma}\right)-L_{\rho_{1}}^{\mathrm{hub}}(\sigma)$ to be sufficiently large. But, since $L_{\rho_{1}}^{\mathrm{hub}}\left(c_{\sigma}\right)$ is bounded from above by

$$
q \cdot \max _{s=0,1, \ldots, t} \max _{x \in \partial \Delta_{s}^{\prime}} 2 \cdot d\left(p_{s}, x\right),
$$

(where we recall that $p_{s}$ is the "center" of the hub $\Delta_{s}$ ) we have the following crude universal upper bound on the amount any such $\sigma$ can save in the hubs compared with its corresponding minimal cycle $c_{\sigma}$ :

$$
L_{\rho_{1}}^{\mathrm{hub}}\left(c_{\sigma}\right)-L_{\rho_{1}}^{\mathrm{hub}}(\sigma) \leq\left\lfloor\frac{\ell_{k}}{\zeta}\right\rfloor \cdot \max _{s=0,1, \ldots, t, t} \max _{x \in \partial \Delta_{s}^{\prime}} 2 \cdot d\left(p_{s}, x\right)
$$

Now, suppose we pick a tubular neighborhood $\mathcal{T}$ of $\mathcal{G}$ contained in $\mathcal{T}^{\prime}$ that is thin enough so that we may choose hubs $\Delta_{0}, \Delta_{1}, \ldots, \Delta_{t}$ satisfying

$$
\left\lfloor\frac{\ell_{k}}{\zeta}\right\rfloor \cdot \max _{s=0,1, \ldots, t} \max _{x \in \partial \Delta_{s}} 2 \cdot d\left(p_{s}, x\right)<\frac{\tilde{\epsilon}}{2}<\tilde{\epsilon} .
$$

Then for any $\sigma$ in $\left(\mathcal{T}, \rho_{1}\right)$ freely homotopic in $\mathcal{T}$ to $c_{\sigma} \in \mathcal{C}$ we have

$$
\begin{aligned}
& L_{\rho_{1}}(\sigma)=L_{\rho_{1}}^{\mathrm{cor}}(\sigma)+L_{\rho_{1}}^{\mathrm{hub}}(\sigma) \\
& \geq L_{\rho_{1}}^{\mathrm{cor}}\left(c_{\sigma}\right)+L_{\rho_{1}}^{\mathrm{hub}}(\sigma) \\
&=L_{\rho_{1}}^{\mathrm{cor}}\left(c_{\sigma}\right)+L_{\rho_{1}}^{\mathrm{hub}}(\sigma)+L_{\rho_{1}}^{\mathrm{hub}}\left(c_{\sigma}\right)-L_{\rho_{1}}^{\mathrm{hub}}\left(c_{\sigma}\right) \\
&=L_{\rho_{1}}\left(c_{\sigma}\right)+L_{\rho_{1}}^{\mathrm{hub}}(\sigma)-L_{\rho_{1}}^{\mathrm{hub}}\left(c_{\sigma}\right) \\
&>L_{\rho_{1}}\left(c_{\sigma}\right)-\tilde{\epsilon} \\
&\left.\geq\left\|\left(a_{c_{\sigma}}, b_{c_{\sigma}}\right)\right\|_{\infty} \text { (by Equation } 2.6\right) \\
&=\left\|\left(a_{\sigma}, b_{\sigma}\right)\right\|_{\infty} .
\end{aligned}
$$


In particular, if $\left(a_{\sigma}, b_{\sigma}\right) \neq\left(a_{i}, b_{i}\right)$ for some $i=1, \ldots, k$, then

$$
L_{\rho_{1}}(\sigma)>\left\|\left(a_{\sigma}, b_{\sigma}\right)\right\|_{\infty} \geq \ell_{k} .
$$

Now let $\sigma$ be a loop in $\left(\mathcal{T}, \rho_{1}\right)$ that is freely homotopic in $\mathcal{T}$ to a minimal cycle $c_{\sigma} \notin \mathcal{C}$. Then $c_{\sigma}$ can be taken to be $\gamma_{i}$ for some $i=2, \ldots, k$ or $c_{\sigma}$ has $q$ edges where $q \geq\left\lfloor\frac{\ell_{k}}{2}\right\rfloor+1$. In the former case, since we are once again interested in a lower bound on the length of $\sigma$ we can assume without loss of generality that $\sigma$ is contained in a (flat) tubular neighborhood $\mathcal{T}_{i} \subset \mathcal{T}$ of $c_{\sigma} \equiv \gamma_{i}$. But, then it follows that since $\rho_{1}$ is flat that we have

$$
L_{\rho_{1}}(\sigma) \geq L_{\rho_{1}}\left(\gamma_{i}\right)=\ell_{i}=\left\|\left(a_{i}, b_{i}\right)\right\| .
$$

In the latter case, we see that $\sigma$ must pass through at least $q$ corridors. Then since each corridor is of length at least $\zeta$ we see

$$
L_{\rho_{1}}(\sigma) \geq \zeta q \geq \zeta \cdot\left(\left\lfloor\frac{\ell_{k}}{\zeta}\right\rfloor+1\right)>\ell_{k} .
$$

In summary, consider the flat regular neighborhood $\left(\mathcal{T}^{\prime}, \rho_{1}\right)$ of $\mathcal{G}$ constructed in Step A and choose a regular neighborhood $\mathcal{T} \subset \mathcal{T}^{\prime}$ of $\mathcal{G}$ with "hubs" $\left\{\Delta_{0}, \Delta_{1}, \ldots, \Delta_{s}\right\}$ satisfying

$$
\operatorname{diam} \Delta_{s} \leq \max _{x \in \partial \Delta_{s}} 2 \cdot d\left(p_{s}, x\right) \leq \Theta \equiv \frac{\tilde{\epsilon}}{2\left\lfloor\frac{\ell_{k}}{\zeta}\right\rfloor},
$$

for each $s=0,1, \ldots, t$, as in Step C. If $\sigma$ is a loop in $\left(\mathcal{T}, \rho_{1}\right)$, then

- $L_{\rho_{1}}(\sigma) \geq \ell_{k}$, if $\left(a_{\sigma}, b_{\sigma}\right) \neq \pm\left(a_{1}, b_{1}\right), \ldots, \pm\left(a_{k}, b_{k}\right)$;

- $L_{\rho_{1}}(\sigma) \geq \ell_{i}$, if $\left(a_{\sigma}, b_{\sigma}\right)= \pm\left(a_{i}, b_{i}\right)$ for some $i=1,2, \ldots, k$;

- $L_{\rho_{1}}\left(\gamma_{i}\right)=\ell_{i}$ for each $i=1,2, \ldots, k$.

Now let $\mathcal{T}_{0} \subset \mathcal{T}_{1} \subset \cdots \subset \mathcal{T}_{4}=\mathcal{T}$ be a collection of properly nested tubular neighborhoods of the graph $\mathcal{G}$ with smooth boundaries such that

(1) $\mathcal{T}=\mathcal{T}_{4}$ admits a metric $\rho_{1}$ as in the lemma;

(2) for each $i=1, \ldots, 4$ and $p, q \in \partial \mathcal{T}_{i}$ we have $d\left(p, \partial \mathcal{T}_{0}\right)=d\left(q, \partial \mathcal{T}_{0}\right)$ where the distance is taken with respect to the metric $\rho_{1}$

and let $\Gamma_{i}=d\left(\partial \mathcal{T}_{i}, \partial \mathcal{T}_{0}\right)$ for each $i=1, \ldots, 4$. Now define the smooth function $r: T^{2} \rightarrow \mathbb{R}$ via

$$
r(x)= \begin{cases}0 & \text { for } x \in \overline{\mathcal{T}_{0}} \\ d_{\rho_{1}}\left(x, \partial \mathcal{T}_{0}\right) & \text { oterhwise }\end{cases}
$$

Now let $\kappa>0$ be such that with respect to $\kappa \rho_{1}$ the distance between $\partial \mathcal{T}_{2}$ and $\partial \mathcal{T}_{1}$ is at least $B$.

Lemma 2.7 (cf. Lemma 5.3 of [DGS]). With the notation and assumptions above there is a Riemannian metric $g$ on $T^{2}$ with the following properties:

(1) $g \succeq g_{0}$ on $T^{2}-\mathcal{T}_{1}$;

(2) $g=g_{0}$ on $T^{2}-\mathcal{T}_{3}$;

(3) $g \succeq \kappa \rho_{1}$ on $\mathcal{T}_{2}-\mathcal{T}_{1}$;

(4) $g=\rho_{1}$ on $\mathcal{T}_{0}$; 
(5) $g \succeq \rho_{1}$ on $\mathcal{T}_{2}$;

where for any metrics $\rho$ and $\tilde{\rho}$ we write $\rho \succeq \tilde{\rho}$ if for all vectors $v$ we have $\rho(v, v) \geq \tilde{\rho}(v, v)$.

Proof of Lemma 2.7. The proof is exactly the same as in [DGS, Lemma 5.3], but we include it for completeness. First, consider the metric $\rho_{2}=g_{0}+\kappa \rho_{1}$ on $\mathcal{T}=\mathcal{T}_{4}$. This metric clearly satisfies $\rho_{2} \succeq g_{0}$ on $\mathcal{T}$. Now let $f_{1}:\left[0, \Gamma_{4}\right] \rightarrow[0,1]$ be a smooth function such that $f_{1}(t)=1$ for $0 \leq t \leq \Gamma_{2}$ and $f_{1}(t)=0$ for $\Gamma_{3} \leq t \leq \Gamma_{4}$. We now define a metric $\widehat{g}$ on $T^{2}$ as follows:

$$
\widehat{g}= \begin{cases}\left(f_{1} \circ r\right) \rho_{2}+\left(1-\left(f_{1} \circ r\right)\right) g_{0} & \text { on } \mathcal{T} \\ g_{0} & \text { on } M-\mathcal{T}_{3}\end{cases}
$$

Then on $T^{2}$ we have $\widehat{g} \succeq g_{0}$ and on $\mathcal{T}_{3}$ we have $\widehat{g}=\rho_{2} \succeq \kappa \rho_{1} \succeq \rho_{1}$. Now let $f_{2}:\left[0, \Gamma_{4}\right] \rightarrow[0,1]$ be a smooth function such that $f_{2}(0)=1$ and $f_{2}(t)=0$ for $\Gamma_{1} \leq t \leq \Gamma_{4}$ and set

$$
g= \begin{cases}\left(f_{2} \circ r\right) \rho_{1}+\left(1-\left(f_{2} \circ r\right)\right) \widehat{g} & \text { on } \mathcal{T} \\ \widehat{g} & \text { on } T^{2}-\mathcal{T}_{1}\end{cases}
$$

Then $g$ satisfies properties (1)-(5).

We now show that any metric $g$ on $T^{2}$ as in Lemma 2.7 has the desired properties. Indeed, let $g$ be such a metric and let $\sigma$ be a homotopically non-trivial curve in $T^{2}$. Then there are three cases.

Case $\mathrm{A}: \operatorname{Im}(\sigma) \subset \mathcal{T}_{2}-\mathcal{T}_{1}$.

Then by Lemma 2.7(1) $g \succeq g_{0}$ on $T^{2}-\mathcal{T}_{1}$, so we see

$$
L_{g}(\sigma) \geq L_{g_{0}}(\sigma) \geq \operatorname{Syst}\left(T^{2}, g_{0}\right)=B \geq \ell_{k} .
$$

Case B: $\operatorname{Im}(\sigma) \cap \mathcal{T}_{1} \neq \emptyset$ and $\operatorname{Im}(\sigma) \cap\left(T^{2}-\mathcal{T}_{2}\right) \neq \emptyset$.

Then, by Lemma 2.7(3) and the way in which $\kappa$ was chosen, we see

$$
L_{g}(\sigma) \geq d_{g}\left(\partial \mathcal{T}_{2}, \partial \mathcal{T}_{1}\right) \geq B \geq \ell_{k} .
$$

Case C: $\operatorname{Im}(\sigma) \subset \mathcal{T}_{2}$.

If $\left(a_{\sigma}, b_{\sigma}\right) \neq\left(a_{1}, b_{1}\right), \ldots,\left(a_{k}, b_{k}\right)$, then using Lemma 2.7(5) and Lemma 2.2 we see

$$
L_{g}(\sigma) \geq L_{\rho_{1}}(\sigma) \geq \ell_{k}
$$

If $\left(a_{\sigma}, b_{\sigma}\right)=\left(a_{i}, b_{i}\right)$ for some $i=1, \ldots, k$ then using (5) and (4) of Lemma 2.7 we see

$$
L_{g}(\sigma) \geq L_{\rho_{1}}(\sigma) \geq \ell_{i} \text {. }
$$

We complete the proof of Theorem 1.3(i) by noting that Lemma 2.7(4) and Lemma 2.2 imply that for each $i=1, \ldots, k L_{g}\left(\gamma_{i}\right)=L_{\rho_{1}}\left(\gamma_{i}\right)=\ell_{i}$.

2.2. The proof of Theorem 1.5. Let $\Psi: \pi_{1}\left(T^{2}, p_{0}\right) \rightarrow H_{1}\left(T^{2} ; \mathbb{Z}\right)$ denote the Hurewicz isomorphism and notice that for any $h \in H_{1}\left(T^{2} ; \mathbb{Z}\right)=H_{1}\left(T^{2} ; \mathbb{Z}\right)_{\mathbb{R}}$ we have $\|h\|_{s}=m_{g}\left(\pi\left(\Psi^{-1}(h)\right)\right)$, where $\pi: \pi_{1}\left(T^{2}\right) \rightarrow \mathcal{F}\left(T^{2}\right)$ is the natural projection of the fundamental group of $T^{2}$ onto the collection of its unoriented free homotopy classes (see p. 3p. It is then apparent that the statement "(ii) implies (i)" is actually a reformulation of Bangert's observation that the stable norm of a 2-torus is strictly convex and the statement "(i) implies (ii)" is equivalent to Theorem 1.3(i). This completes the proof. 


\section{Convergence of the Stable Norms: the Proof of Theorem 1.3(ii)}

In this section we demonstrate that the sequence $\left\langle\|\cdot\|_{j}\right\rangle_{j \in \mathbb{N}}$ of toral stable norms constructed in the previous section converge in the topology of compact convergence to the fixed strictly convex norm $\|\cdot\|_{\infty}$.

Let $g$ be a metric on $T^{2}$ and as in the introduction for each $h \in H_{1}\left(T^{2} ; \mathbb{Z}\right)_{\mathbb{R}}$ let

$$
f(h)=\inf \left\{L_{g}(\sigma): \sigma \text { is a smooth curve representing the class } h\right\} .
$$

Then we have:

(1) $f\left(h_{1}+h_{2}\right) \leq f\left(h_{1}\right)+f\left(h_{2}\right)$ for any $h_{1}, h_{2} \in H_{1}\left(T^{2} ; \mathbb{Z}\right)_{\mathbb{R}}$,

(2) $f(k h)=|k| f(h)$ for any $h \in H_{1}\left(T^{2} ; \mathbb{Z}\right)_{\mathbb{R}}$ and $k \in \mathbb{Z}$; in particular, $f(-h)=f(h)$.

From this we can conclude that for each $h_{1}, h_{2} \in H_{1}\left(T^{2} ; \mathbb{Z}\right)_{\mathbb{R}}$ we have $\left|f\left(h_{1}\right)-f\left(h_{2}\right)\right| \leq$ $f\left(h_{1}-h_{2}\right)=f\left(h_{2}-h_{1}\right)$. It then follows that the associated stable norm $\|\cdot\|_{s}$ will have the property that

$$
\left|\|x\|_{s}-\|y\|_{s}\right| \leq\|x-y\|_{s}
$$

for each $x, y \in H_{1}\left(T^{2} ; \mathbb{R}\right) \simeq \mathbb{R}^{2}$. Now we recall the following basic fact about norms on finite dimensional vector spaces.

Lemma 3.1 (cf. Theorem $7.7[\mathrm{D}]$ ). Let $\phi, \psi: \mathbb{R}^{n} \rightarrow \mathbb{R}$ be norms, then there are constants $0<A \leq B$ such that

$$
A \psi(x) \leq \phi(x) \leq B \psi(x) .
$$

In fact, $A$ and $B$ can be taken to be

$$
A=\frac{\inf \{\phi(x):|x|=1\}}{\left(\sum_{i=1}^{n} \psi\left(\mathbf{e}_{i}\right)^{2}\right)^{1 / 2}}
$$

and

$$
B=\frac{\left(\sum_{i=1}^{n} \phi\left(\mathbf{e}_{i}\right)^{2}\right)^{1 / 2}}{\inf \{\psi(x):|x|=1\}},
$$

where $\left\{\mathbf{e}_{1}, \ldots, \mathbf{e}_{n}\right\}$ is the standard basis for $\mathbb{R}^{n}$ and $|\cdot|$ denotes the standard Euclidean norm with respect to this basis.

Proof. Since $\phi$ is a norm we see that for any $x, y \in \mathbb{R}^{n}$

$$
\phi(x)=\phi(x-y+y) \leq \phi(x-y)+\phi(y) .
$$

It then follows from the fact that $\phi(-v)=\phi(v)$ that

$$
|\phi(x)-\phi(y)| \leq \phi(x-y) .
$$

Now, let $\left\{\mathbf{e}_{1}, \ldots, \mathbf{e}_{n}\right\}$ be the standard basis for $\mathbb{R}^{n}$ and let $x=\sum_{i=1}^{n} x_{i} \mathbf{e}_{i}$ and $y=\sum_{i=1}^{n} y_{i} \mathbf{e}_{i}$ be vectors in $\mathbb{R}^{n}$. Then

$$
\begin{aligned}
|\phi(x)-\phi(y)| & \leq \phi(x-y) \\
& =\phi\left(\sum_{i=1}^{n}\left(x_{i}-y_{i}\right) \mathbf{e}_{i}\right) \\
& \leq \sum_{i=1}^{n}\left|x_{i}-y_{i}\right| \phi\left(\mathbf{e}_{i}\right) \\
& \leq|x-y|\left|\sum_{i=1}^{n} \phi\left(\mathbf{e}_{i}\right) \mathbf{e}_{i}\right| \text { (by the Cauchy-Schwarz Inequality) }
\end{aligned}
$$


where $|\cdot|$ denotes the usual Euclidean norm. Hence, $\phi$ is continuous and taking $y$ to be zero in the equation above we obtain

$$
\phi(x)=|\phi(x)| \leq|x|\left(\sum_{i=1}^{n} \phi\left(\mathbf{e}_{i}\right)^{2}\right),
$$

for each $x \in \mathbb{R}^{n}$. It is then clear that

$$
|x| \inf \{\phi(v):|v|=1\} \leq \phi(x) \leq|x| \sum_{i=1}^{n} \phi\left(\mathbf{e}_{i}\right)^{2},
$$

for any $x \in \mathbb{R}^{n}$. Similarly, we see that $\Psi$ is continuous and that for each $x \in \mathbb{R}^{n}$

$$
|x| \inf \{\psi(v):|v|=1\} \leq \psi(x) \leq|x| \sum_{i=1}^{n} \psi\left(\mathbf{e}_{i}\right)^{2} .
$$

Since $\psi$ is continuous, we see that $\inf \{\psi(v):|v|=1\}$ is positive. Therefore, we may combine Equations 3.2 and 3.3 to establish the claim.

Hence, we see that for any $x, y \in \mathbb{R}^{2}$ and stable norm $\|\cdot\|_{s}$ on the 2-torus we have

$$
\begin{aligned}
\left|\|x\|_{s}-\|y\|_{s}\right| & \leq\|x-y\|_{s} \\
& \leq \frac{\left(\sum_{i=1}^{2}\left\|\mathbf{e}_{i}\right\|_{s}^{2}\right)^{1 / 2}}{\inf \left\{|v|: \sum_{i=1}^{2} v_{i}^{2}=1\right\}}|x-y| \\
& =\left(\sum_{i=1}^{2}\left\|\mathbf{e}_{i}\right\|_{s}^{2}\right)^{1 / 2}|x-y|
\end{aligned}
$$

where throughout $|\cdot|$ denotes the standard Euclidean norm on $\mathbb{R}$ and $\mathbb{R}^{2}$.

We now turn our attention to the sequence of stable norms $\left\langle\|\cdot\|_{j}\right\rangle_{j=1}^{\infty}$ converging to the stable norm $\|\cdot\|_{\infty}$ given by Theorem $1.3(\mathrm{a})$. The $\|\cdot\|_{k}$ 's were constructed so that for each $k \in \mathbb{N}$ we have $\left\|\left(a_{j}, b_{j}\right)\right\|_{k}=\left\|\left(a_{j}, b_{j}\right)\right\|_{\infty}$ for any $1 \leq j \leq k$ and $\left\|\left(a_{j}, b_{j}\right)\right\|_{k} \geq\left\|\left(a_{k}, b_{k}\right)\right\|_{\infty}$ for all $j \geq k+1$. Now fix $N$ large enough so that $(1,0)$ and $(0,1)$ are among the vectors $\left\{\left(a_{j}, b_{j}\right): 1 \leq j \leq N\right\}$. Then we see that for each $j \geq N$ we have $\|(1,0)\|_{j} \equiv\|(1,0)\|_{\infty}$ and $\|(0,1)\|_{j} \equiv\|(0,1)\|_{\infty}$, and it follows from Lemma 3.1 that the constant $B=\left(\|(1,0)\|_{\infty}^{2}+\right.$ $\left.\|(0,1)\|_{\infty}^{2}\right)^{1 / 2}$ satisfies

$$
\left|\|x\|_{j}-\|y\|_{j}\right| \leq B|x-y|
$$

for each $j \geq N$. That is, for $j \geq N$ the stable norms $\|\cdot\|_{j}$ are Lipschitz continuous with the same Lipschitz constant $B$. We now recall the following fact about Lipschitz continuous functions on $\mathbb{R}^{n}$.

Lemma 3.4. Let $\left\langle f_{j}\right\rangle_{j=1}^{\infty}$ be a sequence of functions on $\mathbb{R}^{n}$ for which there exists a constant $C \geq 0$ such that for each $n$

$$
\left|f_{n}(x)-f_{n}(y)\right| \leq C|x-y| \text { for all } x, y \in \mathbb{R}^{n} .
$$

(That is, $\left\langle f_{n}\right\rangle_{n=1}^{\infty}$ is a sequence of Lipschitz continuous functions with the same Lipschitz constant $C$.) If the sequence $\left\langle f_{n}\right\rangle_{n=1}^{\infty}$ converges pointwise to $f: \mathbb{R}^{n} \rightarrow \mathbb{R}$, then $f=\lim _{n \rightarrow \infty} f_{n}$ in the topology of compact convergence. 
Proof. We will show that the $f_{j}$ 's form a uniformly Cauchy sequence on any compact subset $K$ of $\mathbb{R}^{n}$. That is, given $\varepsilon>0$ and compact subset $K \subset \mathbb{R}^{n}$, there is an $N \in \mathbb{N}$ such that $\left|f_{n}(y)-f_{m}(y)\right|<\varepsilon$ for all $n, m \geq N$ and $y \in K$. This implies that $f_{j} \rightarrow f$ uniformly on $K$.

Fix $\varepsilon>0$. Then since $\left\langle f_{j}\right\rangle_{j=1}^{\infty}$ is a sequence of Lipschitz continous functions with the same Lipschitz constant $\mathrm{C}$ we see that for any $j \in \mathbb{N}$ we have $\left|f_{j}(x)-f_{j}(y)\right|<\frac{\varepsilon}{3}$, when $|x-y|<\delta \equiv \frac{\varepsilon}{3 C}$. Now, since the $f_{j}$ 's converge pointwise to $f$ we see that for any $x \in \mathbb{R}^{n}$ there is an $N_{x} \in \mathbb{N}$ such that $\left|f_{n}(x)-f_{m}(x)\right|<\frac{\varepsilon}{3}$ for all $n, m \geq N_{x}$. It then follows that for any $y \in B(x, \delta)$ we have for each $n, m \geq N_{x}$

$$
\begin{aligned}
\left|f_{n}(y)-f_{m}(y)\right| & \leq\left|f_{n}(x)-f_{n}(x)\right|+\left|f_{n}(x)-f_{m}(x)\right|+\left|f_{m}(x)-f_{m}(y)\right| \\
& \leq \frac{\varepsilon}{3}+\frac{\varepsilon}{3}+\frac{\varepsilon}{3} \\
& =\varepsilon
\end{aligned}
$$

Now let $K$ be compact subset of $\mathbb{R}^{n}$, then there are $x_{1}, \ldots, x_{q} \in K$ such that $K \subset$ $\cup_{i=1}^{q} B\left(x_{i}, \delta\right)$. Taking $N=\max \left\{N_{x_{1}}, \ldots N_{x_{q}}\right\}$ it follows that for any $y \in K$ and $n, m \geq N$ we have $\left|f_{n}(y)-f_{m}(y)\right|<\varepsilon$.

Now, by design, the sequence $\left\langle\|\cdot\|_{j}\right\rangle_{j=1}^{\infty}$ converges pointwise to $\|\cdot\|_{\infty}$ on the rational points in $\mathbb{R}^{2}$, but by continuity and denseness we see that they converge pointwise on all of $\mathbb{R}^{2}$ to $\|\cdot\|_{\infty}$. Since for all $j \geq N$, the stable norm $\|\cdot\|_{j}$ is Lipschitz continuous with Lipschitz constant $B=\left(\left\|\mathbf{e}_{1}\right\|_{\infty}^{2}+\left\|\mathbf{e}_{2}\right\|_{\infty}^{2}\right)^{1 / 2}$, it follows from the previous lemma that $\lim _{j \rightarrow \infty}\|\cdot\|_{j}=$ $\|\cdot\|_{\infty}$ in the topology of compact convergence. This completes our argument.

\section{Multiplicities in the minimum MARKED LENGTH SPECTRUM OF TORI}

In this section we will prove Theorem 1.6 which tells us that if $\ell$ is a length of multiplicity $m$ in the minimum length spectrum of $\left(T^{2}, g\right)$, then $n \equiv \# m_{g}^{-1}([0, \ell))$ is bounded from below by a function $f(m)$. That is, if we wish to find a length of multiplicity $m$ in the minimum length spectrum, then we must look beyond the $f(m)$-th term of the sequence. Before proving this theorem it will be useful to recall some facts concerning integer $n$-gons in $\mathbb{R}^{2}$.

An integer $n$-gon is an $n$-gon in $\mathbb{R}^{2}$ whose vertices lie in the lattice $\mathbb{Z}^{2}$. Given an integer $n$-gon $P$, Pick's theorem tells us that the area of the region bounded by $P$, denoted by $A(P)$, can be computed as follows

$$
A(P)=i(P)+\frac{b(P)}{2}-1
$$

where $i(P)$ denotes the number of lattice points in the interior of the region bounded by $P$ and $b(P)$ equals the number of lattice points on the boundary $P$. Now for each $k$ we let $\mathcal{P}_{k}^{+}$ denote the collection of convex integer $k$-gons and set

$$
A(k) \equiv \min \left\{A(P): P \in \mathcal{P}_{k}^{+}\right\} .
$$

Bounds for the function $A(k)$ have been studied for some time. In fact, Andrews was the first to observe that $A(k)$ grows roughly like $k^{3}[\mathrm{~A}]$. Some recent improvements and related results that will be useful in our argument are summarized below. But, first we give a definition.

Definition 4.1. A (bounded) region $R \subset \mathbb{R}^{2}$ is said to be centrally symmetric with respect to $p_{0} \in \mathbb{R}^{2}$, if for any $x_{1} \in R$ there is a point $x_{2} \in R$ such that $p_{0}$ is the midpoint of the line segment joining $x_{1}$ and $x_{2}$. 
Proposition 4.2. The function $A(k)$ enjoys the following properties:

(1) (Rabinowitz, [Rab]) $\frac{1}{8 \pi^{2}}<\frac{A(k)}{k^{3}}<\frac{1}{54}+O(1)$;

(2) (Bárány-Tokushige, [BT, Theorem 1]) $\lim _{k \rightarrow \infty} \frac{A(k)}{k^{3}}$ exists;

(3) (Bárány-Tokushige, [BT, Claim 1]) for every $k$ even, there is a $k$-gon $\widehat{P}_{k}$ that is centrally symmetric with respect to some $(x, y) \in \frac{1}{2} \mathbb{Z}^{2}$ and such that $A(k)=A\left(\widehat{P}_{k}\right)$.

Now for each $k \in \mathbb{N}$ we let

$$
i(k) \equiv \min \{i: \text { there exists a } k \text {-gon with exactly } i \text { interior points }\} \text {. }
$$

Then since any integer $k$-gon contains an inscribed $k$-gon with exactly $k$ lattice points on it, Pick's theorem tells us that

$$
i(k)=A(k)+\frac{2-k}{2},
$$

and we conclude that the problem of finding among convex $k$-gons the least number of interior points is the same as finding the $k$-gon of smallest area. The following is than an immediate consequence of Proposition 4.2 .

Proposition 4.3. The function $i(k)$ enjoys the following properties:

(1) $\frac{1}{8 \pi^{2}}+o(k)<\frac{i(k)}{k^{3}}<\frac{1}{54}+O(k)$;

(2) $\lim _{k \rightarrow \infty} \frac{i(k)}{k^{3}}$ exists;

(3) for every $k$ even, there is an $k$-gon $\widehat{P}_{k}$ that is centrally symmetric about the origin $(0,0)$ and such that $i(k)=i\left(\widehat{P}_{k}\right)$.

With these preliminaries out of the way we may now prove Theorem 1.6 .

Proof of Theorem 1.6. Consider a torus $\left(T^{2}, g\right)$ which has a length $\ell$ of multiplicity $m$ in its minimum length spectrum, and let $\pm\left(a_{1}, b_{1}\right), \ldots, \pm\left(a_{m}, b_{m}\right) \in \mathbb{Z}^{2}$ represent the (not necessarily primitive) unoriented free homotopy classes in $m_{g}^{-1}(\ell)$, and set $n \equiv \# m_{g}^{-1}([0, \ell))$. Then the points $\pm\left(a_{1}, b_{1}\right), \ldots, \pm\left(a_{m}, b_{m}\right)$ determine an integer $2 m$-gon that is centrally symmetric about $(0,0)$ with exactly $2 n-1$ interior points. Now, for each $k \in \mathbb{N}$ we consider the following odd integer

$$
i_{0}^{\text {symm }}(2 k) \equiv\left\{i(Q): Q \in \widehat{P}_{2 k} \text { is centrally symmetric with respect to }(0,0)\right\} .
$$

Then we see that

$$
n \geq f(m) \equiv \frac{i_{0}^{\mathrm{symm}}(2 m)+1}{2} .
$$

Since $i_{0}^{\text {symm }}(2 m) \geq i(2 m)=O\left(m^{3}\right)$, this establishes the first part of the claim.

To see that this inequality is sharp pick $m \in \mathbb{N}$ and let $\widehat{Q}_{2 m}$ be a $2 m$-gon with vertices $\left\{ \pm\left(a_{1}, b_{1}\right), \ldots, \pm\left(a_{m}, b_{m}\right)\right\}$ that is centrally symmetric with respect to $(0,0)$ and such that $i\left(\widehat{Q}_{2 m}\right)=i_{0}^{\text {symm }}(2 m)$. Since every centrally symmetric $2 m$-gon with center $(0,0)$ contains an inscribed centrally symmetric $2 m$-gon with center $(0,0)$ and whose only intersection with $\mathbb{Z}^{2}$ occurs at the $2 m$-vertices, we see that the boundary of $\widehat{Q}_{2 m}$ contains exactly the $2 m$ vertices. Now let $c$ be the boundary of a strictly convex region $B \subset \mathbb{R}^{2}$ that is centrally symmetric with respect to $(0,0)$ and such that the intersection of $c$ with $\mathbb{Z}^{2}$ is precisely the collection of vertices of $\widehat{Q}_{2 m}$. (There are many ways to find such a curve. One way is by replacing each of the segments in $\widehat{Q}_{2 m}$ by convex polynomial arcs such that the resulting tangent vectors at the beginning and the end of the arcs remain outside the resulting shape. 
The centrally symmetric condition is easily met by doing this simultaneously on opposite edges with symmetric arcs.)

Now let $\|\cdot\|_{c}$ be the unique strictly convex norm on $\mathbb{R}^{2}$ such that $c$ is precisely the set of points in $\mathbb{R}^{2}$ with $\|(x, y)\|_{c}=\ell>0$. Then by Theorem 1.3 there is a Riemannian metric $g$ on $T^{2}$ whose stable norm agrees with $\|\cdot\|_{c}$ on the set of $(a, b) \in \mathbb{Z}^{2}$ such that $\|(a, b)\|_{c} \leq \ell$ and has norm strictly larger than $\ell$ for all other lattice points. It follows that the metric $g$ is such that $\ell$ has multiplicity $m=\# m_{g}^{-1}(\ell)$ in the minimum length spectrum and the number of unoriented free homotopy classes for which the shortest geodesic is of length less than $\ell$ is precisely $\# m_{g}^{-1}([0, \ell))=f(m) \equiv \frac{i_{0}^{\text {symm }}(2 m)+1}{2}$.

Proof of Corollary 1.7. The idea here is to take a hyperbolic once punctured or one holed torus, construct a comparable compact smooth closed torus from it, and apply Theorem 1.6. More precisely, for a one-holed torus with geodesic boundary, by glueing in a euclidean hemisphere of the same boundary length, one obtains a closed torus. For a once-punctured torus, one can mimic this construction by first removing a small horocyclic neighborhood of the cusp, of say length 1, and then glueing a euclidean hemisphere of equator length 1. Minimum length geodesics on this torus do not enter the added euclidean hemisphere. To see this, consider an arc of a curve that does cross a hemisphere. The arc has length at least the length of the shortest equator path between the two endpoints of the arc. The new curve obtained by replacing the arc by the equator path is either shorter or of equal length but is no longer smooth and thus cannot be of minimum length. We can conclude that a minimum length geodesic is entirely contained in the hyperbolic part of the torus. As minimum length curves are always simple closed geodesics, the result on minimum length curves on a smooth torus now naturally correspond to simple closed geodesics on the hyperbolic tori. Now Theorem 1.6 asserts that if there are $m$ distinct homotopy classes associated to equal minimum length geodesics, then there are at least $f(m)$ homotopy classes with shorter length representatives and this proves the corollary.

Remark 4.4. We note that in [MR], McShane and Rivin used the stable norm on the homology of a punctured torus to study the asymptotic growth of the number of simple closed geodesics of length less than $\ell$ on a hyperbolic torus.

\section{REFERENCES}

[A] George Andrews, A lower bond for the volume of strictly convex bodies with many boundary lattice points, Trans. Amer. Math. Soc., 106 (1963), 270-279.

[B1] Victor Bangert, Minimal geodesics, Ergodic Theory Dynam. Systems, 10(2):263-286, 1990.

[B2] Victor Bangert, Geodesic rays, Busemann functions and monotone twist maps, Calc. Var., 2: 49-63, 1994.

[BT] Imre Bárány and Norihide Tokushige, The minimum area of convex lattice $n$-gons, Combinatorica, 24 (2004), no. 2, 171-185.

[DGS] Bart de Smit, Ruth Gornet and Craig J. Sutton, Sunada's method and the covering spectrum, J. Differential Geom., to appear.

[D] Harry Dym, Linear Algebra in Action, Graduate Studies in Mathematics, 78. American Mathematical Society, Providence, RI, 2007.

[FHS] Michael Freedman, Joel Hass, and Peter Scott, Closed geodesics on surfaces, Bull. London Math. Soc., 14 (1982), no. 5, 385-391.

[Gr] Misha Gromov, Metric structures for Riemannian and non-Riemannian spaces, Modern Birkhäuser Classics. Birkhäuser, Boston, 2007.

[L] John M. Lee, Introduction to topological manifolds, Springer, Berlin, 2000. 
[MP] Greg McShane and Hugo Parlier, Multiplicities of simple closed geodesics and hypersurfaces in Teichmüller space, Geom. Topl., 12 (2008), 1883-1919.

[MR] Greg McShane and Igor Rivin, A norm on homology on homology of surfaces and counting simple geodesics, Internat. Math. Res. Notices 1995, no. 2, 61-69.

[Rab] S. Rabinowitz, $O\left(n^{3}\right)$ bounds for the area of a convex lattice $n$-gon, Geombinatorics, 2 (1993), 85-88.

[Ran] B. Randol, The length spectrum of a Riemann surface is always of unbounded multiplicity, Proc. Amer. Math. Soc., 78 (1980), no. 3, 455-456.

[Sch] Paul Schmutz-Schaller, Geometry of Riemann surfaces based on closed geodesics, Bull. Amer. Math. Soc., 35 (1998), no. 3, 193-214.

(Eran Makover) Central Connecticut State University, Department of Mathematics, New BRITAIN, CT

E-mail address: makover@ccsu.edu

(Hugo Parlier) Department of Mathematics, University of Fribourg, Switzerland

E-mail address: hugo.parlier@gmail.com

(Craig J. Sutton) Dartmouth College, Department of Mathematics, Hanover, NH 03755

E-mail address: craig.j.sutton@dartmouth.edu 